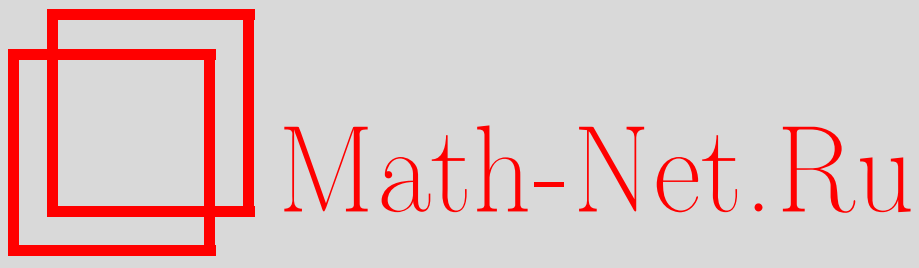

А. Ю. Колесов, Н. Х. Розов, Явление буферности в математической модели генератора ван дер Поля с распределенными параметрами, Изв. РАН. Сер. матем., 2001, том 65, выпуск 3, 67-84

DOI: https://doi.org/10.4213/im336

Использование Общероссийского математического портала Math-Net.Ru подразумевает, что вы прочитали и согласны с пользовательским соглашением

http://www.mathnet.ru/rus/agreement

Параметры загрузки:

IP : 34.227 .88 .159

26 апреля 2023 г., 17:26:26 
УДК 517.926

\author{
А. Ю. Колесов, Н. Х. Розов
}

\title{
Явление буферности в математической модели генератора Ван дер Поля с распределенными параметрами
}

\author{
Для математической модели распределенного генератора Ван дер Поля, пред- \\ ставляющей собой нелинейную краевую задачу гиперболического типа, устана- \\ вливается явление буферности, т.е. существование при подходящем выборе па- \\ раметров любого фиксированного числа устойчивых циклов. \\ Библиография: 22 наименования.
}

\section{Введение}

Будем говорить о феномене буферности, если в системе дифференциальных уравнений с частными производными при подходящем выборе параметров можно гарантировать существование любого конечного числа устойчивых циклов (периодических по времени решений). В случае гиперболических уравнений исследование этого явления по инициативе Ю. С. Колесова начато в [1]. Затем оно было продолжено в работах [2]-[4], из результатов которых следует, что буферность типична для гиперболических краевых задач, причем в некоторых случаях возможна даже гипербуферность [4], т.е. существование сразу счетного числа устойчивых циклов.

С одной стороны, в задачах радиофизики буферность актуальна, например в связи с моделированием процессов памяти и созданием запоминающих ячеек [5]. Но с другой стороны, при конструировании стабильных автогенераторов надо считаться с этим явлением как с негативньм фактором, так как оно может приводить к спонтанным переходам системы с одного периодического режима на другой под влиянием флуктуационных факторов, т.е. к своеобразной форме хаоса. Поэтому представляет существенный интерес описание простейших радиофизических устройств, обладаюших свойством буферности.

Одним из таких физических устройств, как показано теоретически в работах [6], [7] и экспериментально в статье [8], является RCLG-автогенератор с отрезком длинной линии в цепи обратной связи. Другим примером служит автогенератор, содержащий отрезок длинной линии с туннельньм диодом; соответствующий теоретический и экспериментальный анализ проведен в [9], [10].

В настоящей публикации, продолжающей начатый в статьях [6]-[10] цикл исследований явления буферности в задачах радиофизики, рассматривается математическая модель классического генератора Ван дер Поля с распределенными параметрами, представляющая собой систему телеграфных уравнений с нелинейностями как в самих уравнениях, так и в граничных условиях. Показано, что

Работа выполнена при поддержке РФФИ (грант № 99-01-00157). 
при определенных предположениях в такой модели имеет место феномен буферности. Используемый при этом математический аппарат суть метод бесконечномерной нормализации, являющийся специальным вариантом асимптотического метода Крылова-Боголюбова-Митропольского [11] и опирающийся в алгоритмическом плане на метод квазинормальных форм, разработанный Ю. С. Колесовым [12], [13].

\section{§1. Общие свойства краевой задачи}

1.1. Математическая модель. Идея формирования математической модели распределенного генератора Ван дер Поля связана, в первую очередь, с автогенератором, имеющим сосредоточенные параметры и рассмотренным самим Ван дер Полем (см., например, [14], [15]). Напомним, что речь идет о классическом одноламповом генераторе, схема которого представлена на рис. $1, a$; здесь $L, C, R$ - сосредоточенные индуктивность, емкость и активное сопротивление, а $E_{g}$ - напряжение источника, питающего сетку. Относительно лампы предполагается, что анодный ток $i_{a}$ в ней зависит только от ее сеточного напряжения $u_{c}$ по закону

$$
i_{a}=f\left(u_{c}\right)
$$

где нелинейная функция $f$ - характеристика лампы - имеет график, показанный на рис. 1,6 . Здесь величина $i_{a_{0}}$ определяется постоянным сеточным напряжением $u_{c_{0}}$, а точка $A$ соответствует режиму работы лампы при отсутствии колебаний и называется рабочей точкой.

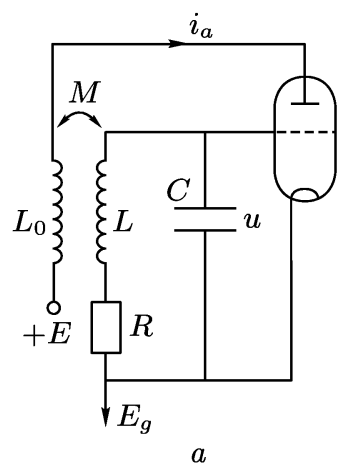

$a$

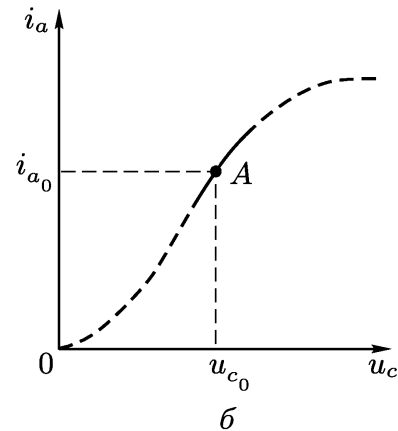

Рис. 1

Как известно, переменная составляющая напряжения в генераторе Ван дер Поля удовлетворяет обыкновенному дифференциальному уравнению

$$
L C \frac{d^{2} u}{d t^{2}}+R C \frac{d u}{d t}+u=M \frac{d i_{a}}{d t}
$$

где $M-$ коэффициент взаимоиндукции между анодной цепью и колебательным контуром в цепи сетки (см. рис. $1, a)$. Аппроксимируем участок характеристики (1.1) в окрестности рабочей точки $A$ кубическим полиномом:

$$
i_{a}=i_{a_{0}}+s_{0} u+s_{1} u^{2}-s_{2} u^{3}
$$


где $s_{0}, s_{2}>0$, а знак $s_{1}$ произволен. Подставив, далее, равенство (1.3) в соотношение (1.2), проведя необходимые нормировки переменных и введя характеристический параметр, придем (см. [15]) к классическому уравнению Ван дер Поля

$$
\ddot{x}+r\left(x^{2}-1\right) \dot{x}+x=0 \text {. }
$$

Отдельно остановимся на физическом смысле правой части уравнения (1.2). Величина $-M d i_{a} / d t$ представляет собой э.д.с. обратной связи, наводимую в колебательном контуре благодаря "индуктивному" воздействию на него анодного тока. Таким образом, через взаимоиндукцию между сеточной (основной) и анодной (вспомогательной) цепями (с индуктивностями $L$ и $L_{0}$ соответственно) происходит "подкачка" энергии в колебательный контур в цепи сетки (в основную цепь), пополняющая часть его энергии, теряемой на сопротивлении $R$.

Допустим теперь, что электрические параметры $L, C, R$ основной цепи равномерно распределены на отрезке проводника длины $l$; тогда получим генератор, изображенньй на рис. 2. Относительно активного элемента (усилителя $У$, имеющего произвольную природу) по-прежнему будем предполагать, что его характеристика задается равенством (1.1).

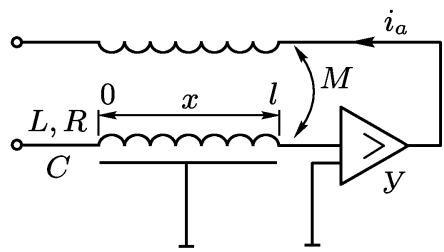

Рис. 2

Пренебрегая распределенной проводимостью $G$, для переменных составляюших напряжения $u$ и тока $i$ в линии $0 \leqslant x \leqslant l$ стандартным образом приходим к системе уравнений

$$
u_{x}=-L i_{t}-R i+M \frac{d i_{a}}{d t}, \quad i_{x}=-C u_{t}
$$

где (ср. с (1.3))

$$
i_{a}=i_{a_{0}}+\left.s_{0} u\right|_{x=l}+\left.s_{1} u^{2}\right|_{x=l}-\left.s_{2} u^{3}\right|_{x=l}
$$

Отметим, что при $M=0$ система переходит в обычную систему телеграфных уравнений, а слагаемое $M d i_{a} / d t$ играет здесь ту же роль, что и аналогичный член в уравнении (1.2), - описьвает обратную связь. Эффект этой обратной связи, очевидно, тот же, что и в классическом генераторе Ван дер Поля, однако теперь “подкачка" энергии в основную цепь осуществляется по всей длине линии $0 \leqslant x \leqslant l$. Ясно и другое: если во вспомогательной цепи сопротивление $R_{0} \ll R$, то естественно пренебречь в ней падением напряжения на сопротивлении $R_{0}$, а значит, можно считать, что величина $i_{a}$ определяется только значением напряжения $u$ на конце линии (см. (1.5)). 
Выполняя в системе (1.4) нормировки $i \sqrt{L / C} \rightarrow i, x / l \rightarrow x, t /(l \sqrt{L C}) \rightarrow t$ и вводя обозначения $\varepsilon=R l \sqrt{C / L}, \beta_{j}=M s_{j} / \sqrt{L C}, j=0,1,2$, переходим к системе уравнений $(t \geqslant 0 ; 0 \leqslant x \leqslant 1)$

$$
u_{t}=-i_{x}, \quad i_{t}=-u_{x}-\varepsilon i+\frac{d}{d t}\left[\left.\beta_{0} u\right|_{x=1}+\left.\beta_{1} u^{2}\right|_{x=1}-\left.\beta_{2} u^{3}\right|_{x=1}\right]
$$

которую, в свою очередь, дополним граничными условиями

$$
\left.i\right|_{x=0}=0,\left.\quad i\right|_{x=1}=0
$$

Заметим, что первое из условий (1.7) - следствие разомкнутости основной цепи на конце $x=0$, а второе вытекает из предполагаемой идеальности усилителя, т.е. наличия у него бесконечно большого входного активного сопротивления.

Описанный автогенератор с распределенными параметрами по физической сути максимально приближен к классическому сосредоточенному генератору Ван дер Поля. Поэтому будем называть его распределенным генератором Ван дер Поля. Краевая задача (1.6), (1.7), представляющая одну из возможных математических моделей этого генератора, была предложена (на основе развитой в работе [16] методики) в заметке [17]. Следует упомянуть и статью [18], где предпринят теоретический анализ модели (1.6), (1.7) и приведены результаты физического эксперимента.

1.2. Разрешимость начальной задачи. Положим

$$
v=f(t)-i, \quad f(t)=\left.\beta_{0} u\right|_{x=1}+\left.\beta_{1} u^{2}\right|_{x=1}-\left.\beta_{2} u^{3}\right|_{x=1}
$$

и перейдем от задачи (1.6), (1.7) к эквивалентной краевой задаче

$$
\begin{gathered}
u_{t}=v_{x}, \quad v_{t}=u_{x}+\varepsilon(f(t)-v) \\
\left.v\right|_{x=0}=\left.v\right|_{x=1}=\left.\beta_{0} u\right|_{x=1}+\left.\beta_{1} u^{2}\right|_{x=1}-\left.\beta_{2} u^{3}\right|_{x=1}
\end{gathered}
$$

В дальнейшем нам потребуется гильбертово пространство $W_{2}^{1}\left([0,1] ; \mathbb{R}^{2}\right)$ со скалярным произведением

$$
(g, h)=\int_{0}^{1}(g(x), h(x)) d x+\int_{0}^{1}\left(g^{\prime}(x), h^{\prime}(x)\right) d x
$$

где $(*, *)$ - обычное скалярное произведение в $\mathbb{R}^{2}$. Обозначим через $\Sigma$ нелинейное многообразие функций $(u(x), v(x)) \in W_{2}^{1}\left([0,1] ; \mathbb{R}^{2}\right)$, удовлетворяющих условиям (1.9) и равенству

$$
\int_{0}^{1} u(x) d x=0
$$

а через $B(r)$ - замкнутый шар в пространстве $W_{2}^{1}\left([0,1] ; \mathbb{R}^{2}\right)$ радиуса $r$ с центром в нуле. 
ТЕОРема 1.1. Пусть в краевых условиях (1.9) коэффициент $\beta_{0} \neq 1$. Тогда по любому $T>0$ можно указать такие достаточно малье $\varepsilon_{0}=\varepsilon_{0}(T)>0$ $u r=r(T)>0$, что при всех $0<\varepsilon \leqslant \varepsilon_{0}$ любое решение $(u(t, x, \varepsilon), v(t, x, \varepsilon))$ краевой задачи (1.8), (1.9) с произвольныцми начальнымми условиями

$$
\left.u\right|_{t=0}=u(x),\left.\quad v\right|_{t=0}=v(x): \quad(u(x), v(x)) \in \Sigma \cap B(r)
$$

одназначно определено на отрезке $|t| \leqslant T$, принадлежит при каждом $t$ многообразию $\Sigma$ и сильно непрерывно по $t$ в метрике $W_{2}^{1}\left([0,1] ; \mathbb{R}^{2}\right)$.

ДокАЗАТЕльСтво. Убедимся сначала в разрешимости задачи (1.8), (1.9), (1.11) при $\varepsilon=0$. В этом случае решение системы (1.8) с начальными условиями (1.11) имеет вид

$$
u(t, x)=\varphi(t+x)+\psi(t-x+1), \quad v(t, x)=\varphi(t+x)-\psi(t-x+1),
$$

где

$$
\varphi(x)=\frac{[u(x)+v(x)]}{2}, \quad \psi(x)=\frac{[u(1-x)-v(1-x)]}{2} .
$$

Подставляя формулы (1.12) в граничные условия (1.9), получим однозначный способ продолжения функций $(1.13)$ с отрезка $0 \leqslant x \leqslant 1$ на всю ось посредством равенств

$$
\varphi(t)-\psi(t+1)=\varphi(t+1)-\psi(t)=H[\varphi(t+1)+\psi(t)]
$$

здесь $H(z)=\beta_{0} z+\beta_{1} z^{2}-\beta_{2} z^{3}$.

Для доказательства разрешимости системы разностных уравнений (1.14) в сторону возрастания $t$ рассмотрим сначала вспомогательное уравнение $\varphi-\psi=$ $H(\varphi+\psi)$ и применим к нему в точке $\varphi=\psi=0$ теорему о неявной функции (ее справедливость обеспечивает условие $\beta_{0} \neq 1$ ). В итоге получим функцию $\varphi=G(\psi)$, $G(0)=0$, определенную на некотором отрезке $|\psi| \leqslant q$, где $q>0$ достаточно мало. Возвращаясь к системе (1.14), заключаем, что при дополнительном условии

$$
|\psi(t)| \leqslant q
$$

она эквивалентна системе явных разностных уравнений

$$
\varphi(t+1)=G(\psi(t)), \quad \psi(t+1)=\varphi(t)-H(G(\psi(t))+\psi(t)) .
$$

Остается заметить, что, во-первых, при подходящей малости радиуса $r$ в условии (1.11) решение $(\varphi(t), \psi(t))$ этой системы однозначно продолжается методом шагов на любой фиксированный отрезок $0 \leqslant t \leqslant T$ (с сохранением неравенства (1.15)); во-вторых, $\varphi(t), \psi(t) \in W_{2}^{1}(0, T)$ в силу того, что начальные функции удовлетворяют условиям согласования

$$
\varphi(0)-\psi(1)=\varphi(1)-\psi(0)=H(\varphi(1)+\psi(0)) .
$$

Ясно, что аналогичные выводы справедливы относительно разрешимости системы (1.14) в сторону убывания $t$. 
Отдельно остановимся на доказательстве справедливости для компоненты $u(t, x)$ решения системы (1.12) равенства (1.10). Из первого уравнения (1.8) и граничных условий (1.9) для функции

$$
m(t)=\int_{0}^{1} u(t, x) d x
$$

получаем уравнение $d m / d t \equiv 0$, а отсюда и из условия $m(0)=0$ (см. (1.10)) заключаем, что $m(t)=0$ при всех $t$.

При $0<\varepsilon \ll 1$ ситуация в принципе не меняется. В этом случае, рассматривая слагаемое $\varepsilon f(t)$ во втором уравнении (1.8) как неоднородность и интегрируя систему (1.8) методом Римана (см., например, [19]), для неизвестных $u, v$ также получаем явные формулы. Подставляя их в краевые условия (1.9), приходим к системе нелинейных интегральных уравнений, разрешимость которой при $0<\varepsilon \ll 1$ вытекает из уже установленной ее разрешимости при $\varepsilon=0$ и теоремы о неявной функции. Теорема доказана.

Из теоремы 1.1 следует, что решения краевой задачи (1.8), (1.9) порождают в фазовом пространстве $\Sigma$ сильно непрерывную по $t$ группу ограниченных нелинейных операторов $U^{t}(h), \quad h=\operatorname{colon}(u(0, x), v(0, x))$. Из способа построения этой группы вытекает, что она гладко (по Фреше) зависит от начального условия $h$ и параметров. А это, в свою очередь, позволяет практически без изменений распространить на краевую задачу (1.8), (1.9) основные положения качественной теории обыкновенных дифференциальных уравнений: теоремы Ляпунова и Андронова-Витта об устойчивости по первому приближению, утверждения об интегральных многообразиях и т.д.

Обратим внимание, что при отказе от условия (1.10) у краевой задачи $(1.8),(1.9)$ появляется однопараметрическое семейство состояний равновесия $\left(u_{0}, H\left(u_{0}\right)\right)$, $-\infty<u_{0}<\infty$. Физический же смысл имеет только одно из них - нулевое. Поэтому становится понятен смысл ограничения (1.10): оно “отсекает" все посторонние положения равновесия. Дополнительный аргумент в пользу принятия этого ограничения состоит и в том, что при учете распределенной проводимости (т.е. при замене первого уравнения (1.8) на $\left.u_{t}=v_{x}-\alpha u, \alpha>0\right)$ для функции (1.16) справедливо уравнение $m^{\prime}+\alpha m=0$, у которого любое решение $m(t)$ стремится к нулю при $t \rightarrow \infty$.

1.3. Линейный анализ. В дальнейшем будем интересоваться периодическими решениями краевой задачи (1.8), (1.9), бифурцируюшими из нулевого состояния равновесия. Поэтому сначала исследуем его на устойчивость.

Рассмотрим характеристическое уравнение

$$
\beta_{0}(1-\operatorname{ch} \mu)+\mu \lambda^{-1} \operatorname{sh} \mu=0, \quad \mu=\sqrt{\lambda^{2}+\varepsilon \lambda},
$$

для определения его спектра устойчивости. Несложный анализ показывает, что, во-первых, при $\varepsilon=\beta_{0}=0$ корни уравнения (1.17) простые, чисто мнимые и исчерпываются равенствами $\lambda= \pm i \omega_{n}, \omega_{n}=n \pi, n=1,2, \ldots$, а отвечающие им решения линеаризованной задачи имеют вид

$$
u=\exp \left( \pm i \omega_{n} t\right) \cos \omega_{n} x, \quad v= \pm i \exp \left( \pm i \omega_{n} t\right) \sin \omega_{n} x
$$


во-вторых, при $0<\varepsilon \ll 1, \beta_{0}=\gamma \varepsilon, \gamma \sim 1$, корни уравнения (1.17) распадаются на две группы: $\lambda_{2 n+1}(\varepsilon), \bar{\lambda}_{2 n+1}(\varepsilon)$ и $\lambda_{2 n+2}(\varepsilon), \bar{\lambda}_{2 n+2}(\varepsilon), n=0,1, \ldots$, допускающие равномерную по $n$ асимптотику

$$
\begin{aligned}
& \lambda_{2 n+1}(\varepsilon)=i \omega_{2 n+1}+\varepsilon(2 \gamma-1 / 2)+O\left(\varepsilon^{2}\right), \\
& \lambda_{2 n+2}(\varepsilon)=i \omega_{2 n+2}-\varepsilon / 2+O\left(\varepsilon^{2}\right) .
\end{aligned}
$$

Из формул (1.18) следует, что нулевое решение задачи (1.8), (1.9) экспоненщиально устойчиво при $\gamma<1 / 4$ и неустойчиво при $\gamma>1 / 4$, причем в последнем случае в полуплоскость $\operatorname{Re} \lambda>0$ переходят сразу все корни (1.18) с нечетными номерами - наблюдается так называемая взрывная неустойчивость. Данное обстоятельство в совокупности с резонансностью частот при $\varepsilon=0$ (в счетном числе) приводит, вообще говоря, к реализации в задаче (1.8), (1.9) разрывных колебаний (см. [17]). Поэтому ниже ее автоколебания будем исследовать при условии

$$
\beta_{0}=\varepsilon / 4+\varepsilon^{2} \gamma
$$

где параметр $\gamma>0$ имеет порядок единицы. Как будет ясно из последующего анализа, диапазон (1.19) изменения параметра $\beta_{0}$ соответствует плавному переходу от устойчивого нулевого состояния равновесия (при $\gamma<0)$ через гладкие автоколебания и явление буферности (при средних $\gamma>0$ ) к разрывным колебаниям (при достаточно больших $\gamma$ ).

Итак, исследованию подлежит краевая задача (ср. с (1.8), (1.9))

$$
\begin{gathered}
u_{t}=v_{x}, \quad v_{t}=u_{x}-\varepsilon v+\varepsilon\left[\left.\left(\varepsilon / 4+\varepsilon^{2} \gamma\right) u\right|_{x=1}-\left.u^{3}\right|_{x=1}\right] \\
\left.v\right|_{x=0}=\left.v\right|_{x=1}=\left.\left(\varepsilon / 4+\varepsilon^{2} \gamma\right) u\right|_{x=1}-\left.u^{3}\right|_{x=1}
\end{gathered}
$$

Равенства $\beta_{2}=1$ (см. (1.9)) добиваемся за счет подходяшей нормировки переменных $u, v$; случай $\beta_{1}=0$ (симметричная характеристика $f$, см. (1.8)) рассматривается лишь для простоты, так как все полученные результаты остаются в силе и при $\beta_{1} \neq 0$.

\section{§2. Метод бесконечной нормализации}

2.1. Построение нормальной формы. Сначала изложим формальный алгоритм построения нормальной формы краевой задачи (1.20), а его обоснование дадим ниже.

Следуя методике Ю. С. Колесова (см. [12], [13]), подставим в задачу (1.20) ряды

$$
\begin{aligned}
& u=\varepsilon u_{0}(t, s, x)+\varepsilon^{2} u_{1}(t, s, \tau, x)+\varepsilon^{3} u_{2}(t, s, \tau, x)+\cdots, \\
& v=\varepsilon v_{0}(t, s, x)+\varepsilon^{2} v_{1}(t, s, \tau, x)+\varepsilon^{3} v_{2}(t, s, \tau, x)+\cdots,
\end{aligned}
$$

где $s=\varepsilon^{2} t, \tau=\varepsilon t$,

$$
\begin{aligned}
& u_{0}=\sum_{n=0}^{\infty}\left[z_{n}(s) \exp \left(i \omega_{2 n+1} t\right)+\bar{z}_{n}(s) \exp \left(-i \omega_{2 n+1} t\right)\right] \cos \omega_{2 n+1} x \\
& v_{0}=i \sum_{n=0}^{\infty}\left[z_{n}(s) \exp \left(i \omega_{2 n+1} t\right)-\bar{z}_{n}(s) \exp \left(-i \omega_{2 n+1} t\right)\right] \sin \omega_{2 n+1} x
\end{aligned}
$$


а комплексные "амплитуды" $z_{n}$ таковы, что сходится ряд с общим членом $\omega_{2 n+1}^{2}\left|z_{n}\right|^{2}$ (в этом случае $u_{0}, v_{0} \in W_{2}^{1}$ по переменной $x$ ). Приравняв затем коэффициенты при $\varepsilon^{2}$ и $\varepsilon^{3}$, для нахождения 2-периодических по $t$ функций $u_{j}, v_{j}, j=1,2$, получим краевые задачи

$$
\begin{gathered}
\frac{\partial u_{1}}{\partial t}=\frac{\partial v_{1}}{\partial x}, \quad \frac{\partial v_{1}}{\partial t}=\frac{\partial u_{1}}{\partial x}-v_{0},\left.\quad v_{1}\right|_{\substack{x=0 \\
x=1}}=\left.\frac{1}{4} u_{0}\right|_{x=1} \\
\frac{\partial u_{2}}{\partial t}=\frac{\partial v_{2}}{\partial x}-\frac{\partial u_{0}}{\partial s}-\frac{\partial u_{1}}{\partial \tau}, \quad \frac{\partial v_{2}}{\partial t}=\frac{\partial u_{2}}{\partial x}-\frac{\partial v_{0}}{\partial s}-\frac{\partial v_{1}}{\partial \tau}-v_{1}+\left.\frac{1}{4} u_{0}\right|_{x=1}, \\
\left.v_{2}\right|_{\substack{x=0 \\
x=1}}=\left.\frac{1}{4} u_{1}\right|_{x=1}+\left.\gamma u_{0}\right|_{x=1}-\left.u_{0}^{3}\right|_{x=1} .
\end{gathered}
$$

Исключая из системы (2.3) переменную $v_{1}$, приходим к краевой задаче

$$
\left(\frac{\partial^{2}}{\partial t^{2}}-\frac{\partial^{2}}{\partial x^{2}}\right) u_{1}=-\frac{\partial u_{0}}{\partial t},\left.\quad \frac{\partial u_{1}}{\partial x}\right|_{\substack{x=0 \\ x=1}}=\left.\frac{1}{4} \frac{d}{d t} u_{0}\right|_{x=1}
$$

решение $u_{1}$ которой имеет вид ряда:

$$
\begin{aligned}
u_{1}= & \sum_{n=0}^{\infty} z_{n} A_{n}(x) \exp \left(i \omega_{2 n+1} t\right)+\bar{z}_{n} \bar{A}_{n}(x) \exp \left(-i \omega_{2 n+1} t\right) \\
& +\sum_{n=0}^{\infty}\left[w_{n}(\tau) \exp \left(i \omega_{2 n+2} t\right)+\bar{w}_{n}(\tau) \exp \left(-i \omega_{2 n+2} t\right)\right] \cos \omega_{2 n+2} x
\end{aligned}
$$

Здесь второе слагаемое - произвольное периодическое решение однородной краевой задачи, отвечающее собственным частотам $\omega_{n}$ с четными номерами, а комплексные "амплитуды" $w_{n}$ таковы, что сходится ряд с общим членом $\omega_{2 n+2}^{2} \cdot\left|w_{n}\right|^{2}$.

Подчеркнем, что разрешимость краевых задач $\left({ }^{\prime}=d / d x\right)$

$$
A_{n}^{\prime \prime}+\omega_{2 n+1}^{2} A_{n}=i \omega_{2 n+1} \cos \omega_{2 n+1} x,\left.\quad A_{n}^{\prime}\right|_{x=1} ^{x=0}=-i \omega_{2 n+1} / 4
$$

для определения коэффициентов $A_{n}(x)$ ряда (2.5) обеспечивается за счет констант, фигурирующих в граничных условиях. Сами же решения $A_{n}(x)$, удовлетворяющие дополнительным условиям $A_{n}(1)=0$, имеют вид

$$
A_{n}(x)=[i(2 x-1) / 4] \sin \omega_{2 n+1} x .
$$

Таким образом, проясняется формальная математическая причина специального выбора (1.19) коэффициента $\beta_{0}$. Учитывая представление (2.5) в первом уравнении (2.3), получим

$$
v_{1}=\left.\frac{1}{4} u_{0}\right|_{x=1}+\int_{0}^{x} \frac{\partial u_{1}}{\partial t}(t, s, \tau, y) d y .
$$

Исходя из задачи (2.4), приходим к краевой задаче

$$
\begin{gathered}
\left(\frac{\partial^{2}}{\partial t^{2}}-\frac{\partial^{2}}{\partial x^{2}}\right) u_{2}=-\frac{\partial u_{1}}{\partial t}-2 \frac{\partial^{2} u_{0}}{\partial t \partial s}-2 \frac{\partial^{2} u_{1}}{\partial t \partial \tau} \\
\left.\frac{\partial u_{2}}{\partial x}\right|_{\substack{x=0 \\
x=1}}=\frac{d}{d t}\left[\left.\frac{1}{4} u_{1}\right|_{x=1}+\left.\gamma u_{0}\right|_{x=1}-\left.u_{0}^{3}\right|_{x=1}\right]
\end{gathered}
$$


решение $u_{2}$ которой определяется в виде ряда:

$$
\begin{aligned}
u_{2}= & \sum_{n=0}^{\infty} B_{n}(x) \exp \left(i \omega_{2 n+1} t\right)+\bar{B}_{n}(x) \exp \left(-i \omega_{2 n+1} t\right) \\
& +\sum_{n=0}^{\infty} C_{n}(x) \exp \left(i \omega_{2 n+2} t\right)+\bar{C}_{n}(x) \exp \left(-i \omega_{2 n+2} t\right) .
\end{aligned}
$$

Здесь $B_{n}(x), C_{n}(x)$ - решения краевых задач

$$
\begin{gathered}
B_{n}^{\prime \prime}+\omega_{2 n+1}^{2} B_{n}=i \omega_{2 n+1} z_{n} A_{n}(x)+2 i \omega_{2 n+1} \frac{d z_{n}}{d s} \cos \omega_{2 n+1} x, \\
\left.B_{n}^{\prime}\right|_{\substack{x=0 \\
x=1}}=-i \omega_{2 n+1} f_{n} \\
C_{n}^{\prime \prime}+\omega_{2 n+2}^{2} C_{n}=i \omega_{2 n+2}\left(2 \frac{d w_{n}}{d \tau}+w_{n}\right) \cos \omega_{2 n+2} x,\left.\quad C_{n}^{\prime}\right|_{\substack{x=0 \\
x=1}}=\frac{i \omega_{2 n+2}}{4} w_{n},
\end{gathered}
$$

a $f_{n}-$ коэффищиент при $\exp \left(i \omega_{2 n+1} y\right)$ ряда $\Phi$ урье функции $\gamma \theta-\theta^{3}$, где

$$
\theta(s, y)=\sum_{n=0}^{\infty} z_{n}(s) \exp \left(i \omega_{2 n+1} y\right)+\bar{z}_{n}(s) \exp \left(-i \omega_{2 n+1} y\right)
$$

Условия разрешимости задач (2.8) и (2.9) приводят к равенствам

$$
\frac{d z_{n}}{d s}=\frac{i z_{n}}{8 \omega_{2 n+1}}+2 f_{n}, \quad \frac{d w_{n}}{d \tau}=-\frac{w_{n}}{2}, \quad n=0,1, \ldots
$$

Учитывая их в правых частях уравнений $(2.8),(2.9)$ и решая получившиеся в итоге краевые задачи, убеждаемся в том, что

$$
\begin{gathered}
B_{n}=\frac{z_{n}}{8}(x-1)\left(x-\frac{1}{4}\right) \cos \omega_{2 n+1} x-\left(\frac{z_{n}}{4 \omega_{2 n+1}}-2 i f_{n}\right)\left(x-\frac{1}{2}\right) \sin \omega_{2 n+1} x, \\
C_{n}=\left(i w_{n} / 4\right) \sin \omega_{2 n+2} x .
\end{gathered}
$$

И наконец, из первого уравнения (2.4) определяем

$$
\begin{aligned}
v_{2}= & \int_{0}^{x} \frac{\partial u_{2}}{\partial t}(t, s, \tau, y) d y+\sum_{n=0}^{\infty}\left[\left(\frac{i z_{n}}{8 \omega_{2 n+1}^{2}}+\frac{2 f_{n}}{\omega_{2 n+1}}\right) \exp \left(i \omega_{2 n+1} t\right)\right. \\
& \left.+\left(-\frac{i \bar{z}_{n}}{8 \omega_{2 n+1}^{2}}+\frac{2 \bar{f}_{n}}{\omega_{2 n+1}}\right) \exp \left(-i \omega_{2 n+1} t\right)\right] \sin \omega_{2 n+1} x+\left.\left(\gamma u_{0}-u_{0}^{3}\right)\right|_{x=1}- \\
& -\frac{1}{4} \sum_{n=0}^{\infty}\left[w_{n} \exp \left(i \omega_{2 n+2} t\right)+\bar{w}_{n} \exp \left(-i \omega_{2 n+2} t\right)\right] \frac{1}{\omega_{2 n+2}} \sin \omega_{2 n+2} x
\end{aligned}
$$

Введем в рассмотрение векторы

$$
z=\operatorname{colon}\left(z_{0}, \bar{z}_{0}, z_{1}, \bar{z}_{1}, \ldots\right), \quad w=\operatorname{colon}\left(w_{0}, \bar{w}_{0}, w_{1}, \bar{w}_{1}, \ldots\right)
$$


принадлежашие пространству $l_{2}^{1}$; оно представляет собой дискретный аналог пространства $W_{2}^{1}$ и состоит из векторов вида $(2.13)$, для которых конечна норма

$$
\|z\|=\left(\sum_{n=0}^{\infty} \omega_{2 n+1}^{2}\left|z_{n}\right|^{2}\right)^{1 / 2} .
$$

Определим, далее, ограниченный нелинейный оператор $F: l_{2}^{1} \rightarrow l_{2}^{1}$ равенством

$$
F(z)=\text { colon }\left(\frac{i z_{0}}{8 \omega_{1}}+2 f_{0},-\frac{i \bar{z}_{0}}{8 \omega_{1}}+2 \bar{f}_{0}, \ldots, \frac{i z_{n}}{8 \omega_{2 n+1}}+2 f_{n}, \ldots\right) .
$$

Наконец, переходя в уравнениях (2.11) к единому времени $s$, получим систему дифференциальных уравнений в $l_{2}^{1} \times l_{2}^{1}$ :

$$
\frac{d z}{d s}=F(z), \quad \varepsilon \frac{d w}{d s}=-\frac{w}{2} .
$$

Система (2.15) как раз и является интересуюшей нас "укороченной" нормальной формой краевой задачи (1.20). Отметим также, что она имеет, очевидно, экспоненциально орбитально устойчивое бесконечномерное инвариантное многообразие $w=0$, движения на котором описываются уравнением

$$
\frac{d z}{d s}=F(z) .
$$

Для анализа последнего нам потребуется гильбертово пространство $E$ антипериодических с периодом 1 функций $\theta(y)$ класса $W_{2}^{1}$.

Из способа определения фигурируюших в равенстве (2.14) коэффициентов $f_{n}=$ $f_{n}(z)$ вытекает, что счетная система (2.16) "сворачивается" в краевую задачу

$$
\frac{\partial \theta}{\partial s}=\frac{1}{8} L \theta+2 \gamma \theta-2 \theta^{3}, \quad \theta(s, y+1) \equiv-\theta(s, y)
$$

для функции (2.10). Здесь $L$ - ограниченный линейньй оператор в $E$, задаюшийся формулой

$$
L \theta=\sum_{n=0}^{\infty} \frac{i}{\omega_{2 n+1}} z_{n} \exp \left(i \omega_{2 n+1} y\right)-\frac{i}{\omega_{2 n+1}} \bar{z}_{n} \exp \left(-i \omega_{2 n+1} y\right)
$$

Дифференцируя уравнение (2.17) по у и учитывая, что $\frac{d}{d y}(L \theta)=-\theta$, приходим к гиперболической краевой задаче

$$
\frac{\partial^{2} \theta}{\partial s \partial y}=-\frac{1}{8} \theta+\left[2 \gamma-6 \theta^{2}\right] \frac{\partial \theta}{\partial y}, \quad \theta(s, y+1) \equiv-\theta(s, y) .
$$

Сформулируем теперь стандартное утверждение о соответствии между грубыми периодическими решениями краевой задачи (1.20) и ее нормальной формы (2.18). 
Теорема 2.1. Пусть краевая задача (2.18) имеет периодическое решение $\theta=\theta_{0}(\xi), \quad \xi=\sigma_{0} s+y, \quad \sigma_{0} \neq 0$, әкспоненциально орбитально устойчивое или дихотомичное (в метрике фазового пространства Е). Тогда в краевой задаче (1.20) при всех достаточно мальх $\varepsilon>0$ ему отвечает иикл той жее устойчивости с асимптотическим представлением (2.1), где при определении коэффициентов следует считать

$$
z_{n}(s)=\theta_{n}^{0} \exp \left(i \omega_{2 n+1} \sigma_{0} s\right), \quad w_{n}=0, \quad n=0,1, \ldots
$$

здесь $\theta_{n}^{0}, \bar{\theta}_{n}^{0}$ - коэффициенты Фурье функции $\theta_{0}(\xi)$ по системе $\exp \left( \pm i \omega_{2 n+1} \xi\right)$, $n=0,1, \ldots$

2.2. Обоснование метода. Из отрезков рядов (2.1) сконструируем замену переменных, действующую из $l_{2}^{1} \times l_{2}^{1}$ в $\Sigma$ и приводящую задачу $(1.20)$ к нормальной форме. Проведем также анализ полученной нормальной формы и обоснуем, в частности, утверждение теоремы 2.1.

При нахождении интересующей нас замены переменных будем считать, что в построенных вьше отрезках рядов (2.1) векторы (2.13) произвольно фиксированны и не зависят от $s$ и $\tau$ соответственно. Тогда получим функции

$$
u=\varepsilon u_{0}(t, x)+\varepsilon^{2} u_{1}(t, x)+\varepsilon^{3} u_{2}(t, x), \quad v=\varepsilon v_{0}(t, x)+\varepsilon^{2} v_{1}(t, x)+\varepsilon^{3} v_{2}(t, x),
$$

которые удовлетворяют граничным условиям задачи (1.20) с точностью до $\varepsilon^{4}$ по невязке. Поэтому для того, чтобы удовлетворить этим граничным условиям точно, перейдем от $(2.20)$ к равенствам

$$
\begin{aligned}
u= & \varepsilon u_{0}(t, x)+\varepsilon^{2} u_{1}(t, x)+\varepsilon^{3} u_{2}(t, x)+\Delta_{1}(t+(x-1), \varepsilon) \\
& +\Delta_{1}(t-(x-1), \varepsilon)+\Delta_{2}(t+(x-1), \varepsilon)-\Delta_{2}(t-(x-1), \varepsilon), \\
v= & \varepsilon v_{0}(t, x)+\varepsilon^{2} v_{1}(t, x)+\varepsilon^{3} v_{2}(t, x)+\Delta_{1}(t+(x-1), \varepsilon) \\
& -\Delta_{1}(t-(x-1), \varepsilon)+\Delta_{2}(t+(x-1), \varepsilon)+\Delta_{2}(t-(x-1), \varepsilon),
\end{aligned}
$$

где подлежат определению функции

$$
\Delta_{1}(t, \varepsilon), \Delta_{2}(t, \varepsilon): \quad \Delta_{1}(t+1, \varepsilon) \equiv-\Delta_{1}(t, \varepsilon), \quad \Delta_{2}(t+1, \varepsilon) \equiv \Delta_{2}(t, \varepsilon) .
$$

Подставляя формулы (2.21) в граничные условия задачи (1.20) и учитывая, что (см. $(2.2),(2.5)-(2.7),(2.12))$

$$
\left.u\right|_{x=1}=-\varepsilon a(t)+\varepsilon^{2} b(t)+2 \Delta_{1}(t, \varepsilon),
$$

где

$$
\begin{aligned}
& a(t)=\sum_{n=0}^{\infty} z_{n} \exp \left(i \omega_{2 n+1} t\right)+\bar{z}_{n} \exp \left(-i \omega_{2 n+1} t\right) \\
& b(t)=\sum_{n=0}^{\infty} w_{n} \exp \left(i \omega_{2 n+2} t\right)+\bar{w}_{n} \exp \left(-i \omega_{2 n+2} t\right)
\end{aligned}
$$


для нахождения функций (2.22) приходим к уравнениям

$$
\begin{gathered}
\left(\varepsilon+4 \gamma \varepsilon^{2}\right) \Delta_{1}+6 \varepsilon^{5} a b^{2}-12\left(\varepsilon^{2} a^{2}+\varepsilon^{4} b^{2}\right) \Delta_{1}+24 \varepsilon a \Delta_{1}^{2}-16 \Delta_{1}^{3}=0 \\
2 \Delta_{2}-\varepsilon^{4} \gamma b+3 \varepsilon^{4} a^{2} b+\varepsilon^{6} b^{3}-12 \varepsilon^{3} a b \Delta_{1}+12 \varepsilon^{2} b \Delta_{1}^{2}=0 .
\end{gathered}
$$

Несложный их анализ приводит к выводу, что функции (2.22) определяются однозначно, причем в метрике пространства $W_{2}^{1}(0,1)$ для них справедливы асимптотические представления

$$
\Delta_{1}(t, \varepsilon)=-\varepsilon^{4} 6 a(t) b^{2}(t)+O\left(\varepsilon^{5}\right), \quad \Delta_{2}(t, \varepsilon)=\varepsilon^{4}\left(\frac{\gamma b(t)}{2}-\frac{3}{2} a^{2}(t) b(t)\right)+O\left(\varepsilon^{5}\right) .
$$

Нам потребуется следующий факт относительно функций (2.22) и фигурирующих в (2.8) коэффициентов $f_{n}=f_{n}(z), n=0,1, \ldots$ : при заменах

$$
z_{n} \rightarrow z_{n} \exp \left(i \omega_{2 n+1} t_{0}\right), \quad w_{n} \rightarrow w_{n} \exp \left(i \omega_{2 n+2} t_{0}\right), \quad n=0,1, \ldots,
$$

где $t_{0} \in \mathbb{R}$ произвольно фиксировано, они переходят соответственно в

$$
\Delta_{1}\left(t+t_{0}, \varepsilon\right), \quad \Delta_{2}\left(t+t_{0}, \varepsilon\right), \quad f_{n}(z) \exp \left(i \omega_{2 n+1} t_{0}\right) .
$$

Для доказательства достаточно заметить, что при заменах (2.24) функции $a(t)$, $b(t)($ см. $(2.23))$ переходят в $a\left(t+t_{0}\right), b\left(t+t_{0}\right)$.

На заключительном этапе построения нормализующего преобразования выполним в равенствах (2.21) замены

$$
z_{n} \exp \left(i \omega_{2 n+1} t\right) \rightarrow z_{n}, \quad w_{n} \exp \left(i \omega_{2 n+2} t\right) \rightarrow w_{n}, \quad n=0,1, \ldots
$$

Из установленного только что факта и приведенных выше явных формул для $u_{j}, v_{j}, j=0,1,2$, вытекает, что результат замен (2.26) есть вектор-функция $h=$ colon $(u, v)$, задающаяся равенством

$$
h=\varepsilon h_{0}(z, x)+\varepsilon^{2} h_{1}(z, w, x)+\varepsilon^{3} h_{2}(z, w, x)+\Omega(z, w, x, \varepsilon),
$$

где

$$
\begin{gathered}
h_{j}=\operatorname{colon}\left(\left.u_{j}\right|_{t=0},\left.v_{j}\right|_{t=0}\right), \quad j=0,1,2, \\
\Omega=\operatorname{colon}\left(\Delta_{1}(x-1, \varepsilon)+\Delta_{1}(1-x, \varepsilon)+\Delta_{2}(x-1, \varepsilon)-\Delta_{2}(1-x, \varepsilon),\right. \\
\left.\Delta_{1}(x-1, \varepsilon)-\Delta_{1}(1-x, \varepsilon)+\Delta_{2}(x-1, \varepsilon)+\Delta_{2}(1-x, \varepsilon)\right) .
\end{gathered}
$$

Равенство (2.27) доставляет нам искомое нормализующее преобразование. Действительно, оно индуцирует нелинейный оператор, действующий из $l_{2}^{1} \times l_{2}^{1}$ в $\Sigma$. Кроме того, из способа построения всех входящих в равенства (2.21) функций следует (см., например, аналогичное место в работе [20]), что результатом вьполнения замены (2.27) в исходной задаче (1.20) является система в $l_{2}^{1} \times l_{2}^{1}$ :

$$
\begin{aligned}
\dot{z} & =\Lambda_{0} z+\varepsilon^{2} F(z)+\varepsilon^{3} \Phi(z, w, \varepsilon), \\
\dot{w} & =\Lambda_{1} w-\varepsilon w / 2+\varepsilon^{2} \Psi(z, w, \varepsilon), \quad \cdot=d / d t,
\end{aligned}
$$


где оператор $F$ задается равенством (2.14),

$$
\begin{aligned}
\Lambda_{0} z & =\operatorname{colon}\left(i \omega_{1} z_{0},-i \omega_{1} \bar{z}_{0}, \ldots, i \omega_{2 n+1} z_{n},-i \omega_{2 n+1} \bar{z}_{n}, \ldots\right) \\
\Lambda_{1} w & =\operatorname{colon}\left(i \omega_{2} w_{0},-i \omega_{2} \bar{w}_{0}, \ldots, i \omega_{2 n+2} w_{n},-i \omega_{2 n+2} \bar{w}_{n}, \ldots\right)
\end{aligned}
$$

а операторы $\Phi(*, *, \varepsilon), \Psi(*, *, \varepsilon): l_{2}^{1} \times l_{2}^{1} \rightarrow l_{2}^{1}$, и их производные по Фреше любого фиксированного порядка ограничены равномерно по $\varepsilon$ на любом ограниченном множестве из $l_{2}^{1} \times l_{2}^{1}$.

В заключение проведем некоторый анализ нормальной формы (2.28). С этой целью рассмотрим отвечающую ей “укороченную” нормальную форму

$$
\dot{z}=\Lambda_{0} z+\varepsilon^{2} F(z), \quad \dot{w}=\Lambda_{1} w-\varepsilon w / 2
$$

и выполним в ней замены, обратные к (2.26). В результате (см. (2.24), (2.25)) приходим к аналогичной (2.15) системе

$$
\dot{z}=\varepsilon^{2} F(z), \quad \dot{w}=-\varepsilon w / 2,
$$

которая в силу условий теоремы 2.1 имеет периодическое решение (2.19) (при $s=$ $\left.\varepsilon^{2} t\right)$, экспоненциально орбитально устойчивое или дихотомичное. Поэтому периодическое решение с теми же свойствами устойчивости, близкое к

$$
z_{n}=\theta_{n}^{0} \exp i \omega_{2 n+1}\left(1+\sigma_{0} \varepsilon^{2}\right) t, \quad w_{n}=0, \quad n=0,1,2, \ldots,
$$

будет иметь (в силу более высокого порядка малости соответствующих остатков $\left.\varepsilon^{3} \Phi, \varepsilon^{2} \Psi\right)$ и система (2.28). Теорема 2.1 доказана.

\section{§ 3. Динамические свойства нормальной формы}

3.1. Гармоническая буферность. Выполняя в краевой задаче (2.18) подходящие нормировки переменных $s, y, \theta$ и заменяя $s$ на $t$, а $y$ на $x$, приведем ее к виду

$$
\theta_{t x}=\theta+\lambda\left(1-\theta^{2}\right) \theta_{x}, \quad \theta(t, x+1) \equiv-\theta(t, x)
$$

где $\lambda=16 \gamma>0$. Напомним, что краевая задача (3.1) была получена в работе [7] как модель другого RCLG-автогенератора. Там же были исследованы вопросы сушествования и устойчивости ее периодических решений. Поэтому здесь мы ограничимся лишь кратким обзором теоретических результатов из работы [7].

В силу теоремы 2.1 особый интерес представляют периодические решения краевой задачи (3.1) типа бегущих волн:

$$
\theta=g(y), \quad y=\sigma t-x, \quad \sigma>0
$$

Будем изучать их динамику при увеличении параметра $\lambda$.

Подставляя в задачу (3.1) формулу (3.2), для определения функции $g=g(y)$, $g(y+1) \equiv-g(y)$, приходим к уравнению

$$
\sigma g^{\prime \prime}+\lambda\left(g^{2}-1\right) g^{\prime}+g=0, \quad '=d / d y,
$$


которое после замены $y / \sqrt{\sigma} \rightarrow y$ преобразуется к виду

$$
g^{\prime \prime}+r\left(g^{2}-1\right) g^{\prime}+g=0
$$

где $r=\lambda / \sqrt{\sigma}$. Заметим, что (3.4) - это классическое уравнение Ван дер Поля, которое при всех $r>0$ имеет (см., например, [21]) единственное периодическое решение $g=g(y, r)$ периода $T=T(r)$ (антипериодическое решение периода $T / 2$ ), причем достаточно гладкие по совокупности переменных функции $g(y, r), T(r)$ таковы, что (см. [15])

$$
\begin{gathered}
g(y, 0)=\exp (i y)+\exp (-i y), \\
T(0)=2 \pi, \quad T^{\prime}(0)=0, \\
T(r)=r T_{0}+r^{-1 / 3} T_{1}+O\left(r^{-1} \ln r\right), \\
T^{\prime}(r)=T_{0}-\frac{1}{3} r^{-4 / 3} T_{1}+O\left(r^{-2} \ln r\right), \quad r \rightarrow \infty, \\
T_{0}=3-2 \ln 2, \quad T_{1}=7,01 \ldots
\end{gathered}
$$

Нас интересует периодическое решение уравнения (3.4) периода $2 / \sqrt{\sigma}$. Поэтому для определения неизвестного параметра $r$ получим уравнение

$$
\varphi(r)=\frac{2}{\lambda}, \quad \varphi(r) \equiv \frac{T(r)}{r} .
$$

При $\lambda \ll 1$, умножая левую и правую части этого уравнения на $\lambda r$ и применяя к получившемуся уравнению в точке $\lambda=0, r=0$ теорему о неявной функции, однозначно определим его решение

$$
r=r_{0}(\lambda): r_{0}(0)=0, \quad r_{0}^{\prime}(0)=\pi, \quad r_{0}^{\prime \prime}(0)=0
$$

Вспоминая, далее, о связи уравнения (3.4) с исходной задачей (3.1), заключаем, что последняя имеет периодическое решение

$$
\begin{gathered}
\theta=g_{0}(y, \lambda), \quad y=\sigma_{0}(\lambda) t-x \\
\sigma_{0}(\lambda)=\left[\lambda / r_{0}(\lambda)\right]^{2}, \quad g_{0}(y, \lambda)=g\left(y / \sqrt{\sigma_{0}(\lambda)}, r_{0}(\lambda)\right) .
\end{gathered}
$$

Заметим также, что наряду с периодическим решением (3.8) у задачи (3.1) при $\lambda \ll 1$ сушествуют и другие периодические решения, получаюшиеся из решения (3.8) с помощью принщипа подобия:

$$
\begin{gathered}
\theta=g_{n}(y, \lambda), \quad y=\sigma_{n}(\lambda) t-x, \quad n=0,1, \ldots \\
g_{n}=g_{0}((2 n+1) y,(2 n+1) \lambda), \quad \sigma_{n}(\lambda)=\sigma_{0}((2 n+1) \lambda) /(2 n+1)^{2} .
\end{gathered}
$$


ТЕорема 3.1. По любому натуральному $N$ можно указать такое достаточно малое $\bar{\lambda}_{N}>0$, что при всех $0<\lambda \leqslant \bar{\lambda}_{N}$ краевая задача (3.1) имеет әкспоненциально орбитально устойчивые периодические решения (3.9) с номерами $n=0,1, \ldots, N$.

Сушествование решения уже доказано. На доказательстве же его устойчивости не останавливаемся, отсылая к работе [7].

Подчеркнем, что согласно определению теорема 3.1 описывает явление буферности, реализуюшееся в краевой задаче (3.1) при $\lambda \ll 1$. А так как в этом случае любое периодическое решение (3.9) с фиксированньм номером $n$ по форме близко к гармоническому, то уместно говорить о гармонической буферности.

3.2. Градиентные катастрофы. Для того чтобы проследить за эволюцией периодического решения (3.8) при увеличении параметра $\lambda$, отметим некоторые свойства функции $\varphi(r)$ (см. (3.6)). Во-первых, из равенств (3.5) вытекает, что

$$
\varphi(r) \sim 2 \pi / r, \quad r \rightarrow+0 ; \quad \varphi(r) \rightarrow T_{0}, \quad r \rightarrow \infty .
$$

Во-вторых, справедливо неравенство

$$
\varphi^{\prime}(r)=\left[r T^{\prime}(r)-T(r)\right] / r^{2}<0, \quad r>0
$$

(при $r \ll 1$ и $r \gg 1$ оно также вытекает из равенств (3.5), а при "средних" значениях $r$ подтверждается численной проверкой).

Из указанных свойств функции $\varphi(r)$ следует, что корень (3.7) уравнения (3.6), а значит, и периодическое решение (3.8) непрерывно продолжаются по параметру $\lambda$ на интервал (см. (3.5)) $0<\lambda<\lambda_{0}, \lambda_{0}=2 /(3-2 \ln 2)$. Рассмотрим, далее, циклы (3.9), получающиеся из (3.8) с помощью принщипа подобия (3.10). Из способа их построения вытекает, что каждый из них существует в "ячейке" $0<\lambda<\lambda_{n}$, $\lambda_{n}=\lambda_{0} /(2 n+1), n=0,1, \ldots$

Итак, при увеличении параметра $\lambda$ кравая задача (3.1) имеет следующую динамику. При малых $\lambda$ наблюдается явление буферности: существует асимптотически много циклов $(3.9)$ с номерами $0 \leqslant n<\left(\lambda_{0} / \lambda-1\right) / 2$, причем можно гарантировать устойчивость любого фиксированного их числа. Далее, каждый цикл (3.9) с фиксированным номером $n$, близкий к гармоническому при $\lambda \ll 1$, с увеличением $\lambda$ усложняется по форме, при $0<\lambda_{n}-\lambda \ll 1$ становится релаксационным (в этом случае является малым множитель $\sigma$ перед старшей производной в уравнении (3.3)), при $\lambda=\lambda_{n}$ - разрывным, а при $\lambda>\lambda_{n}$ - исчезает. И наконец, при $\lambda>\lambda_{0}$ вымирают все циклы (3.9).

Отметим, что способ исчезновения цикла (3.9) при прохождении $\lambda$ через значение $\lambda_{n}$ уместно назвать градиентной катастрофой в силу того, что при $\lambda \rightarrow$ $\lambda_{n}-0 C^{1}$-норма этого цикла стремится к бесконечности, в то время как его $C$-норма остается ограниченной. (Ранее этот феномен для периодических решений типа бегущих волн изучался в статье [22].)

Вопрос об аттракторах краевой задачи (3.1) при $\lambda>\lambda_{0}$ остается открытым. Наиболее вероятньм здесь представляется следующее: все ее решения $\theta(t, x) \not \equiv 0$ при $t \rightarrow \infty$, оставаясь ограниченными в $C$-норме, стремятся к разрывным функциям. 

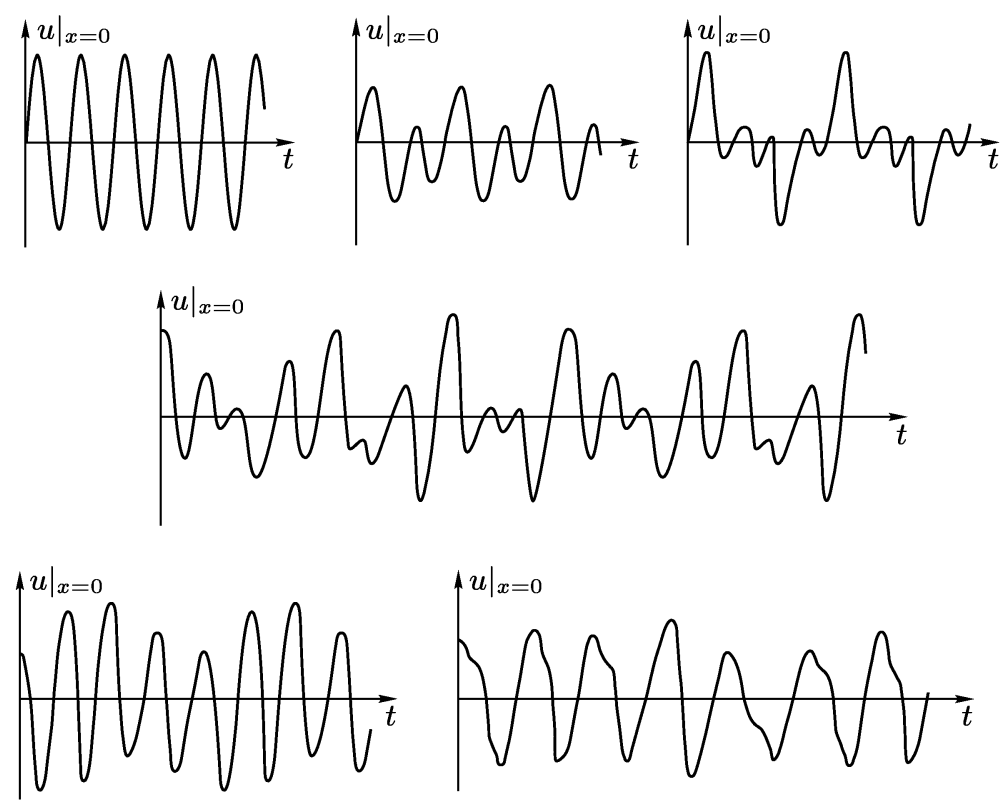

Рис. 3.

В заключение укажем, что в силу установленного в $\S 2$ соответствия между периодическими решениями нормальной формы (3.1) и исходной краевой задачи (1.20) последняя имеет по параметру $\gamma$ аналогичную динамику.

3.3. Результаты эксперимента. Для экспериментальной проверки полученных теоретических результатов В. $\Phi$. Камбуловым был собран автогенератор, блок-схема которого показана на рис. 2. Основная цепь была выполнена в виде прямого соленоида с индуктивностью $L=55.2 \mathrm{м}$ н. Распределенная емкость $C$ между обмоткой катушки и алюминиевой основой составляла 87.7 п $\Phi$, а активное сопротивление $R$ линии равнялось 540 Ом. Следовательно, малый параметр $\varepsilon$ принимал значение 0.022 . Укажем, что диаметр $d$ соленоида равнялся $0.048 \mathrm{M}$, его длина $l_{0}$ составляла $0.429 \mathrm{M}$, толшина $\Delta$ использованного провода равнялась $0.00016 \mathrm{M}$, а обшая длина $l$ всего проводника была $404.32 \mathrm{~m}$.

Вспомогательная цепь была вьполнена в виде прямого соленоида, намотанного на основную цепь. Ее геометрические параметры соответствовали значениям $d=$ $0.054 \mathrm{M}, l_{0}=0.417 \mathrm{M}, \Delta=4 \cdot 10^{-4} \mathrm{M}, l=176.86 \mathrm{~m} ;$ активное сопротивление $R_{0}$ равнялось 17 Ом. Поскольку $R_{0} / R=0.031 \ll 1$, то падением напряжения на $R_{0}$ можно было пренебречь. Таким образом, имела место ситуация, описываемая краевой задачей (1.6), (1.7).

Широкополосный усилитель с входным и выходным активными сопротивлениями, равными соответственно 50 МОм и 75 Ом, обладал равномерным коэффициентом усиления в диапазоне частот от $100 \mathrm{k}$ ц до $120 \mathrm{MГц,} \mathrm{т.е.} \mathrm{по} \mathrm{своим} \mathrm{характе-}$ ристикам был близок к идеальному (его принципиальная схема приведена в [8]). Коэффициент усиления изменялся от 1 до 30 , начальные условия для напряжения и тока задавались различным образом, в том числе и с использованием источни- 
ка синусоидальных колебаний. Периодические режимы, реализующиеся в сконструированном автогенераторе, снимались с выхода усилителя и наблюдались на экране осциллографа.

В результате поставленных экспериментов в некотором диапозоне изменения коэффищиента усиления обнаружено шесть различных сосуществуюших циклов, показанных на рис. 3. Особо подчеркнем, что каждый из них удавалось “вызвать" на экран осциллографа только за счет задания подходящих начальных условий (при фиксированных прочих параметрах), т.е. согласно определению наблюдалось явление буферности. Можно предположить, что запас потенциально реализующихся в рассматриваемом автогенераторе периодических режимов значительно шире. Трудность же их обнаружения связана в первую очередь с узостью области притяжения каждого отдельно взятого режима и с узостью области параметров, в которой имеет место само явление буферности.

Подводя итог проведенному теоретическому и экспериментальному анализу, сформулируем ряд выводов. Во-первых, отличие рассмотренного "распределенного" автогенератора от классического "сосредоточенного" генератора Ван дер Поля весьма условно, так как реально в любом генераторе параметры всегда распределены. Следовательно, можно считать, что в классической задаче установлен новый феномен - явление буферности. Во-вторых, применительно к рассматриваемой ситуации получил дальнейшее развитие метод квазинормальных форм Ю. С. Колесова (см. [12], [13]). Точнее говоря, удалось показать, что при определенных условиях квазинормальная форма является обычной нормальной формой, т.е. исходная краевая задача приводится к ней с помошью некоторой корректной замены переменных. В-третьих, придан дополнительный смысл модельной краевой задаче (3.1), ранее появившейся в работе [7] при анализе RCLG-автогенератора с отрезком длинной линии в цепи обратной связи.

Считаем своим долгом выразить искреннюю благодарность В.Ф. Камбулову за информацию о физической постановке задачи и ее математической модели (1.6), (1.7), а также за предоставленные нам результаты физических экспериментов со сконструированным им автогенератором.

\section{Список литературы}

1. Колесов А.Ю., Колесов Ю. С. Бифуркация автоколебаний сингулярно возмущенного волнового уравнения // ДАН СССР. 1990. Т. 315. № 12. С. 281-283.

2. Колесов А. Ю. Устойчивость автоколебаний телеграфного уравнения, бифурцирующих из состояния равновесия // Матем. заметки. 1992. Т. 51. № 2. С. 59-65.

3. Колесов А. Ю., Розов Н.X. Построение периодических решений уравнения типа Буссинеска с помощью метода квазинормальных форм // Фундамент. и прикл. матем. 1995. T. 1. №1. C. 207-220.

4. Колесов А. Ю. Существование счетного числа устойчивых циклов в средах с дисперсией // Изв. РАН. Сер. матем. 1995. Т. 59. № 3. С. 141-158.

5. Уткин Г. М. Метод медленно меняющихся стоячих волн в теории распределенных автогенераторов // Радиотехника и электроника. 1969. Т. 2. С. 267-276.

6. Камбулов B. Ф., Колесов А. Ю. О явлении буферности в одной резонансной гиперболической краевой задаче из радиофизики // Матем. сб. 1995. Т. 186. № 7. С. 77-96.

7. Камбулов B. Ф., Колесов А.Ю. Об одном модельном гиперболическом уравнении, возникающем в радиофизике // Матем. моделирование. 1996. Т. 8. № 1. С. 93-102. 
8. Камбулов В. Ф., Колесов А. Ю. О специфике генерируемых колебаний в RCLG-автогенераторе с малым затуханием в цепи обратной связи // Радиотехника и электроника. 1997. T. 42. № 8. C. 1019-1024.

9. Камбулов В. Ф., Колесов А. Ю. О явлении буферности в длинной линии с туннельным диодом // Докл. РАН. 1997. Т. 355. № 8. С. 744-746.

10. Камбулов В.Ф., Колесов А.Ю., Розов Н. Х. Теоретический и экспериментальный анализ феномена буферности в длинной линии с туннельным диодом // Дифференц. уравнения. 1997. Т. 33. № 5. С. 638-645.

11. Боголюбов Н. Н., Митропольский Ю. А. Асимптотические методы в теории нелинейных колебаний. М.: Наука, 1974.

12. Колесов Ю.С. Бифуркация инвариантных торов параболических систем с малой диффузией // Матем. сб. 1993. Т. 184. № 3. С. 121-136.

13. Колесов А. Ю. Асимптотика и устойчивость нелинейных параметрических колебаний сингулярно возмущенного телеграфнного уравнения // Матем. сб. 1995. Т. 186. № 10. C. $57-72$.

14. Андронов A. А., Витm A.А., Хайкин С. Э. Теория колебаний. М.: Физматгиз, 1959.

15. Мищенко E. Ф., Розов H.X. Дифференциальные уравнения с малым параметром и релаксационные колебания. М.: Наука, 1995.

16. Колесов Ю.С. Математическая теория RC-генераторов с распределенными параметрами в цепи обратной связи // Дифференц. уравн. и их применение. № 2. Вильнюс: Ин-т физики и математики АН Лит. ССР, 1971. С. 3-68.

17. Камбулов $B . \Phi$. Резонансность как источник релаксационных колебаний в системах телеграфиых уравнений // Докл. РАН. 1994. Т. 334. № 5. С. 569-570.

18. Камбулов В. Ф. Модель распределенного автогенератора Ван-дер-Поля // Радиотехника и электроника. 1997. Т. 42. № 9. С. 1121-1124.

19. Кошлянов Н.С., Глинер Э.Б., Смирнов М. М. Уравнения в частных производных математической физики. М.: Высшая школа, 1970.

20. Колесов А. Ю. Параметрические колебания решений телеграфного уравнения с умеренно малой диффузией // Сиб. матем. журн. 1992. Т. 33. №6. С. 79-86.

21. Рейссиг Р., Сансоне Г., Конти Р. Качественная теория нелинейных дифференциальных уравнений. М.: Наука, 1974.

22. Колесов А. Ю. Релаксационные циклы нелинейного волнового уравнения, гладко зависящего от параметров // Докл. РАН. 1995. Т. 341. № 2. С. 158-160.

Поступило в редакцию 26.IV. 2000 